\title{
The Dynamics and Impacts of Programming Languages in Varying Technologies
}

\author{
Ngozi Kingsley-Opara \\ Computer Science Department, \\ Ignatius Ajuru University of Education, \\ Port Harcourt Nigeria
}

\author{
Nathaniel Akpofure Ojekudo \\ Computer Science Department, \\ Ignatius Ajuru University of Education, \\ Port Harcourt Nigeria
}

\begin{abstract}
The problem-solving capability in programming and programming technique is what has been utilised to tackle most of the ever arising computing related challenges in our society. Its ability to perform specific task is what has been harnessed in solving technology related issues which have metamorphosed into varying technologies in our today's society. Significantly, programming with its techniques and languages has contributed immensely to solving societal problems. In the struggle to achieve the objective of this study, the use of explanatory and exploratory method of research is adopted. On this note the individual technology supported by various programming languages are identified.
\end{abstract}

\section{Keywords}

Programming Language, Technology, Instructional Codes, Computing

\section{INTRODUCTION}

Programming as a set of instructional codes that output what can be used to perform specific tasks has been utilized in various areas covering various works of life to solve problemoriented tasks. So the importance and relevance of programming in the science of computing technology cannot be overemphasized. In the basic definition of a computer that states it as an electronic device that accepts data and processes it according to pre-determined instructions, it can be drawn that the pre-determined instruction is coming from the instructional codes of a programming language. So it can be seen that one can't talk about computing technology without mentioning the programming language which is pivotal to the technology. The development and advancement of these instructions have metamorphosed into many application areas of the industry in their varying technologies. This in essence has emerged into embracing the new generation technologies of our time. The act of programming is majorly to create software for computers used in solving problems. The constant development of the language is targeted at problemoriented areas [1].

There would not have been many technologies in the world of information technology today if not that the emergence of various programming languages has been utilized in solving various problems in the society. From this, one can also see the real impact of the use of programming languages to write the instructions that have helped in solving societal problems in the present time more than what the society use to have. Sequel to this therefore, there are going to be considerations of some programming languages and their various applications which have given rise to the varying technologies available today. The languages under considerations include Python, Java, JavaScript, Ruby, C, C\#, C++, PHP, Swift and Go.
The observance of the ever-rising technological growth is quite interesting but there is a need to know what is responsible to this great fit. The dissection of the problem solving technique in programming is needed to showcase various areas they have been used to promote the technological advancement.It can be seen clearly that the technology of computing powered by writing programming instructional codes customized to meet a particular need in the society has gradually entered into solving problems in many works of life. This has led to the existing varying technologies supported by the computing technology of the present digital era.

\subsection{Aim and Objectives}

This study aims to show how techniques of programming language have helped in the various developments of some apps that have transformed into various technologies. This can be demonstrated through the following objectives:

- Provide an understanding of what programming entails

- Explain different programming languages and corresponding areas of relevance

- Explore impacts of programming and their consequent contribution to the varying technology

\subsection{Scope}

The coverage areas of this study are on concept of programming, some application areas and their corresponding technology they support.

\subsection{Significance}

The relevance of this study is for the audience to understand and appreciate how programming has contributed immensely to solving societal problems and subsequent contributions to technological advancement in this digital era

\section{REVIEW OF RELATED WORKS}

The standard created by the IEEE (Institute of Electrical and Electronics Engineers) and ACM (Association for Computing Machinery) has helped in the all-encompassing ways of creating applications that can fit into varying technologies. In this regard [1] noted that there are various standards for computers and programming. Many international organizations such as IEEE and ACMhave been seriously involved in the development of computers and programming standards. In 2005, the Computer Standards were published which include related terms and application of Computer Engineering, Computer Science, Information Systems, Information Technology, and Software Engineering. As a result, after the creation of the standards,the systems are able to interrelate and inter-cooperate by fitting into other technologies hence making the various programming languages create various technologies on the foundation of the same concept.The programming language has advanced into 
the technology of data science and data management according to[2]who affirmed that this advancement in technology for instance noted thatthe new technology of processing data has replaced the old and normal method of keeping record. The underlying instructional codes in programming language have been utilized significantly in the software development that targeted problem-solving in the area of mobile learning technology, which facilitates the improvement of vocabulary and writing skill, and ultimately provide interactive and collaborative privileges. The constant evolution and advancement of technology can be seen as aligning mutually with the corresponding languages used in achieving such technology. It is noted in [3] that the banking sectors for instance who invest primarily in the security of their system, Java happens to have the security features needed to achieve the security objectives and consequently is used in developing banking applications.

Some programming Languages have been with us for decades but due to the fact that the technology they support had not evolved as at the time of their creation, not much was talked about in such programming languages. Python for instance is an example of such a language. Aaccordingto [4], Python has well been around over 3 decades but at that time, the technology of artificial intelligence and machine learning had not been popularly embraced as it is today. So the relevance of Python can now been seenas the technology has evolved more.

Given the prominent role programming plays in meeting the trend in the evolving technology of the present time, the programming language is supposed to be treated as a fundamental tool for problem-solving in society. Incidentallyfor scholars in various learning institutions, it is used as a studying and analyzing tool. In computer science, there are advanced concepts that need to be understood which come from the prominent role that programming language plays. Therefore, it is very important to ensure programming language is one of the key courses for computer science scholars as this will pave more ways for technological advancement[5].

The developing and evolving technology is really playing a significant role in modern society and programming language being one of the major factors that contribute immensely to this role is termed a world changer in the computing industry. To this effect, [6] affirmed that modern society has been really impacted by the visible contributions made by the development of modern technology. Consequently, there is an impact of scientific knowledge across various areas of society.

The entrance and impact of programming language on human capital development via learning gave rise to carrying out a survey to determine the impact and effect of digital literacy in learning and development. This literacy hasbeen seen toimprove the rate and quality of teaching andlearningwhichwill be useful in the basic institutions of learning [7].Further research into the impact of programming language in other areas led [8] to conduct a meta-analytical review of the impact of programming languages on the energy consumption of mobile devices of which he concludes that he cannot reject null hypothesis.

The people of an information society need timely information update which is a primary goal of such a society. To achieve this, there is a creation of a communication environment upon which there is a conveyance of knowledge and ideas to the public. That is, in scientific innovation, programming is seen to have played a role that determines the way people of this society acquire their knowledge [9].

\section{METHODOLOGY}

In this study, a strong need to know the extent to which programming technique and its languages have impacted to the ever growing technologies is identified. The use of explanatory and exploratory research methodology is therefore adopted which helped in identifying the various technologies and their supporting programming languages. Afterwards, a research of information which involves the use of web resources, in-print material, journal and other search engines are employed in carrying out this study. Supporting diagrams are captured to clarify some points.

\section{PROGRAMMING LANGUAGES AND THE VARYING TECHNOLOGIES}

The world has evolved into an information age leading into an information society whose major objective is to provide the information needs of the society. To bring an effective and target-oriented result into this concept of information and technology system, the programming technology as a process of sequentiallyand algorithmically writing of instructional codes of computer has consequently advanced to meet with the trend of events in the modern society. Obviously programming has got many languages that played prominent roles in solving societal problems hence leading to the advancement in the technology of the present day. This is to say that the benefits of computing technology have been employed into many works of life and career system thereby playing a pivotal role in today's world as almost every work of life is actually depending on the computing technology to thrive better. Note worthily, the programming language power is characterized by the algorithm, task varieties and number. Sequel to this is a brief discussion of some programming languages and the impacts they have created coupled with their consequential leading to the varying and advancing of the technology as captured below.

\subsection{Python}

One of the primary features of Python is that it is a high-level language. Accordingly as earlier stated in[4], Python has well been around for over 3 decades now. It is used in developing web and game applications, very vital in the technology of machine learning together with that of artificial intelligence and data science.Data mining and visualization coupled with desktop Graphical User Interface (GUI)are not left out in the application area of Python. Other application areas of Python include web scraping and applications, Computer-Aided Design (CAD), audio and video application. Now it can be seen that this language has been around for a time now but was not that popular then as it is now. Due to the trend in technology that has found its way in the Artificial Intelligence (AI),Data Science, and Data Visualization technologies, the relevance and availability of the instructional codes and techniques that would drive this technology are found in Python programming. Since then, this language has played a prominent role in driving and having an impact on AI technology.This is a language considered to be beginnerfriendly because the codes are simple to understand in the course of learning. Also, it is a general-purpose language that has very human-friendly instructional codes.Python language has the power to expand internet apps, information analysis, user interfaces, and for additional frameworks are also accessible for these tasks. Python is employed by larger firms principally which will appraise with the capability that can evaluate huge data sets [10]. 


\subsection{Java}

Java is a high level language as well. It is most secured and due to this security nature and feature, the technology of banking development in mobile banking, banking apps, server apps in the financial service industry that require strong security features are developed with Java. In fact according to [11], it is a secure, high performance, and portable objectoriented programming language. It is widely used in the technology of android development, Internet of Things (IoT), cloud computing, games or desktop application, hence it is a general purpose programming language and tends to reveal a lot of things that are behind the scene. Java is used for mobilebased applications, enterprise-level purpose, for making desktop applications, and for establishing Android apps on tablets and Smartphones as noted in [10].

\subsection{JavaScript}

This is a programming languagethat has a client end and a server end, that is to say that it works in a client browser (that is, the browser that a user loads as opposed to working and interacting with the server behind the scene). It is a high-level prototype-based object-oriented programming language. It is widely used for front-end development. Java Script is a programming language for web development and the technology the web development offers, of which some are found in the technology of image and graphic development, very useful in the graphic industry. The beauty about this is that JavaScript is paired with HTML and CSS (a world wide web technology) to make up the front-end of the trio of the programming language in buildinga website (or any application), that is, to build the front-end of the website or any other application. Java Script can as well be used as a back-end language.

\subsection{Ruby}

This is a high-level language as well as object-oriented. New timers or beginners find it very interactive and friendly and one can build things very quickly using the framework of "Reels". It is also a general-purpose programming language used in robotics, networking, web application development, security, and system administration. Used for simulation by National Aeronautics and Space Administration(NASA).

\section{5. $\mathrm{C}$}

This is an old and one of the most widely used programming languages having being around for decades. It is a structureoriented, middle-level programming language and often used for low-level applications, very widely used for developing device drivers - very powerful and was the inspiration behind many high-level programming languages. It is used to develop system applications that are integrated into the operating system as such as Windows, Unix, and Linus. It is also used for system programming, in the technology of artificial intelligence, industrial automation technology, computer graphic technology, space research, image processing in game development.

\subsection{C\#}

This is a language that supports multiple strategies or paradigms and that is to say that it supports multiple types of logic. It is developed about two decades ago by Microsoft Corporation as part of its .NET initiative. This is similar to C, $\mathrm{C}++$, Java and it is widely used for developing widows, applications, and games. C\# is also a general-purpose programming language enclosing object-oriented, imperative, functional, generic, event-driven, and component-oriented programming styles (DLLs and Assemblies). It was designed by Andres Hejlsberg (the creator of Turbo Pascal), developed by Microsoft, and first released in July 2000. C\# was developed especially for the .NET platform with the main goal of providing a simple, powerful, and strongly typed programming language allowing programmers to quickly build a wide range of applications for the .NET platform [12]. Similar to Java, it is also an object-oriented programming language that comes with an extensive class library and supports exception handling, multiple types of polymorphism, and separation of interfaces from implementations [11].

\section{7. $\mathrm{C}++$}

This language here can be said to be middle-level and at the same time is object-oriented. In fact, it is an extension of C programming language while having an extraction and still keeping the power of that $\mathrm{C}$ foundation. It is also a generalpurpose programming language that is widely used to work system with application software drivers, client-server applications.One of the benefits of $\mathrm{C}++$ is that when it is being used to create object-oriented code, the programmer has good object-oriented features to facilitate maintainability [11].

\subsection{PHP}

This is designed to create dynamic web pages that work well with databases and supports more than one language strategy or paradigm. It is widely used for web application development, server-side scripting, and command-line scripting. With the assistance of PHP, one can enlarge a web app quickly and effortlessly. The run time employed by the compiler to execute the logic is few seconds and the memory used is $2.57 \mathrm{mb}$. PHP is the actual foundation of many strong content management systems [10].

\subsection{Swift}

Swift is multiple paradigmsof Apple used to develop apps for IOS (iPhoneoperating system, that is, a mobile operating system) and OS. It is created to preserve the concept of or objective of $\mathrm{C}$ which is the other language one can use to create apps for Apple. It was also created to be safer so that it catches up bugsin a more eloquent manner and easy way, andalso good for building native apps for iPhones.

\subsection{GO}

This is a property of Google and it is developed to enhance theproductivity of programming. To achieve this fit, the following features were captured in the design:readability, usability like Python or JavaScript, static timing and runtime efficiencies like $\mathrm{C}++$, and high-performance networking and multiprocessing. It is a general-purpose multi-paradigm Programming language, very concise, single, and safe meaning that because the syntax is so simple, it is so much easy to catch and get rid of any potential bug. It is designed to solve Google size problems that are very complex. It is a universal programming language, so it is useful for everyone: From the development of ordinary programs to the development of complex systems, this language can be used. In this sense, it is closer to $\mathrm{C}$ and $\mathrm{C}++$. Go incorporates modern functions, removes unnecessary data from memory, and provides parallelism[9].

\section{IMPACT AND CONTRIBUTIONS TO THE VARYING TECHNOLOGY}

The world is actually evolving occasioned by the evolving technology. In attempt to solving the inevitable human problems, the programming language dynamically has found 
its way into the non-computing areaswith the objective of rendering solutions to the existing problems.

\subsection{Biomedical Engineering:}

In the health sector for instance, [13] demonstrated that MATLAB (Matrix Laboratory, a type of programming language) in conjunction with ECG (Electrocardiogram) Signal can be used to detect bradycardia condition (that is, a condition of the heart to determine its health status as being slower than a normal heart beat) in a patient. This is to say that after collecting the required data from a patient, MATLAB can be used to extract signal sample of the heartbeat rate of thepatient and with the various samples gathered, it can be determined if the patient heartbeat rate is normal or abnormal.

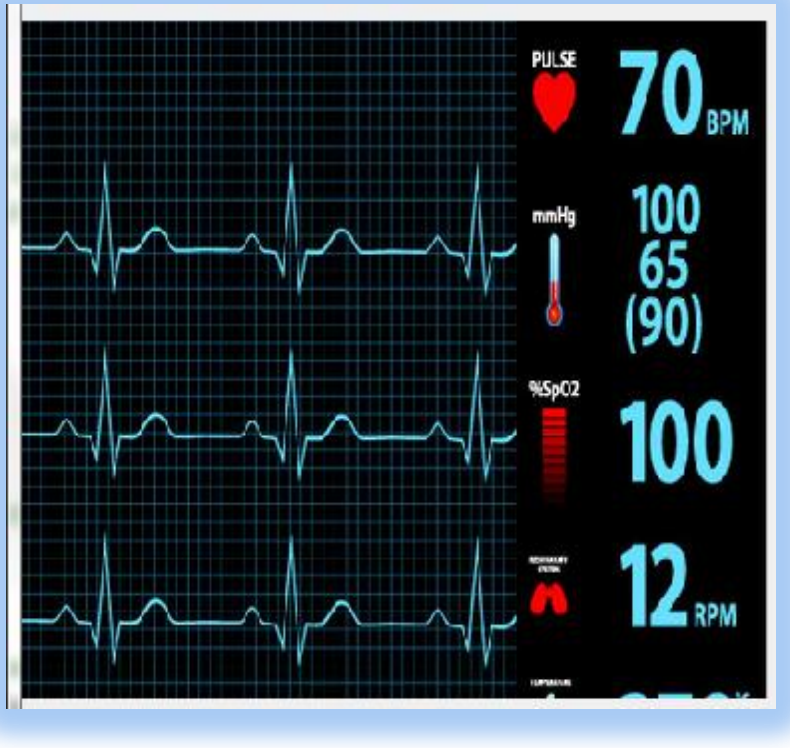

Fig 1: Signal Processing with MATLAB

\subsection{Machine Learning and Artificial Intelligence (AI):}

Machine learning is a technique whereby a computer is trained or taught how to make accurate predictions when it is fed with data. As the data is stored, the computer can learn based on the past experience of the stored data. The more data is fed for the training, the better the expected result. Alternatively, an algorithm can be written for the computer to train and learn by itself. This technique is used in speech recognition, that is, speech-to-text used in generating captions for videos. The act of employing human intelligence in some smart machines to perform certain tasks is what artificial intelligence (AI) offers. "Generally, the term "AI" is used when a machine simulates functions that humans associate with other human minds, such as learning and problem solving" [14]. Python as a programming language is very useful in this technology.

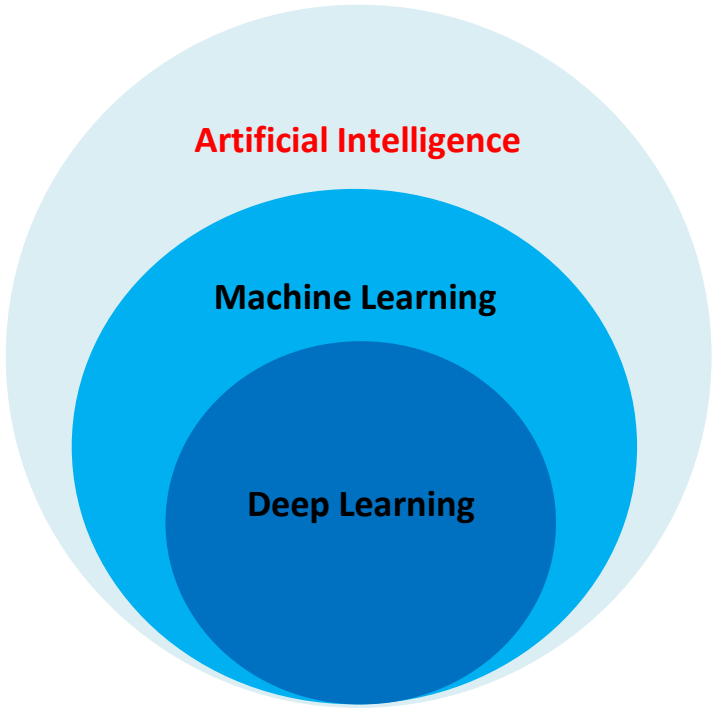

Fig 2: Interphase of AI with Machine Learning

\subsection{Data Science and Data Visualization}

The present era is an era of technology where there is an appreciation of information displayed visually using various kinds of graphs. Also, information displayed visually is better explained and understood than when it is in its raw and text format. This istherefore an age where more data are required and acquired for various daily tasks and activities. Now the more the accumulation of raw data, the more complex theretrieval of information becomes. So the art and technique of manipulating, interpreting, and bringing out useful information from the raw data is where data science that deals with the ability to discover and reveal patterns and correlations that may not have been noticed comes into play. The required information is better understood when it is related and displayed visually such as using graphs and histograms. To affirm the above information, [15]said thus "Data visualization is important to group together many data points, to understand relationships in data, debate questions in real time and more quickly decide where to focus research. It helps data scientists to identify hidden data patterns and how it is processed".

\subsection{Business Applications}

One can rightly guess why there is a boom in the e-commerce industry, it is because programming language with its instructional codes has directed how client can do trading without being physically present at the business premises or shopping mall. Taking instances from the big-time online shops such as Jumia, Alibaba, Konga, and the rest as ascertained by [16], one can see the convenience of procuring goods and services from one's comfort zone irrespective of miles and distance apart. This e-commerce breakthrough is a result of the advancement in the Enterprise Resource Planning (ERP) arm of the database system, altogether digital and affiliate marketing, another arm of e-commerce is thriving upon this breakthrough. 


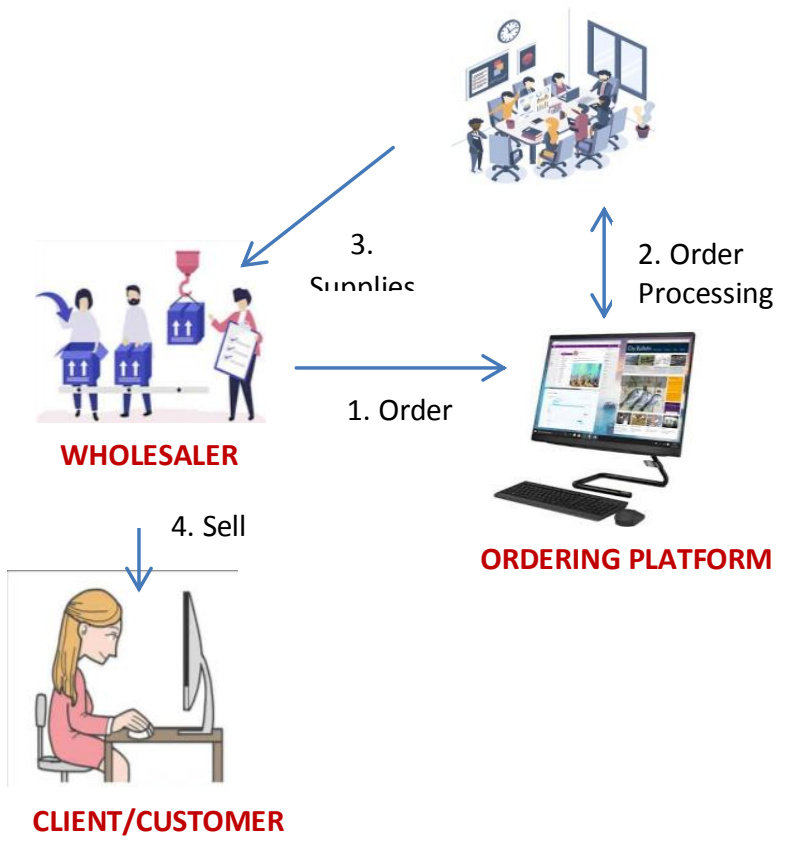

Fig 3: E-Commerce Platform

\subsection{Computer-Aided Design Applications}

Inthe architectural industriesprogramming technique has solved problems in their architectural designs by using Computer-Aided Design (CAD) software. One beautiful thing about this technique is that depending on the version of the software used, the 3 and 4-dimensional view of the design can be captured[3].The beauty of this technology is that one can have afull view of inside and outside of the architectural structure of work before the real-life physical structure. Python is very useful in the development of CAD applications used for the architectural design work.

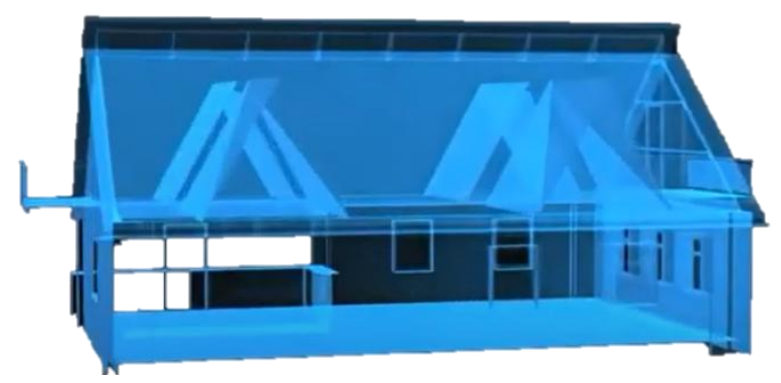

Fig 4:A sample of a front view of a 3-dimentional CAD design

\subsection{Mobile Applications}

The instructional codes in Java find much relevance in mobile applications and it is also evident that Kotlinand Android Studio software are compatible. The android mobile application are actually developed using Java and Kotlin, the combination of HTMl, JavaScript and CSS can equally be used to develop native apps which are thetypes directly installed into the smart devices such as phone and may require no connectivity of internet to work.

\subsection{Big Data Technologies}

Today's daily activities give rise to the acquisition of large and complex data that need analysis by a way of the systematic extraction of information. This technology built on Java is used in managing big and complex data set. There are limitations in the traditional system of data processing which the big data technology designed to break down complex data sets can handle with ease[15]. The prominent role of Java in this technology has made Hadoop, (a notable technology) what it is today.

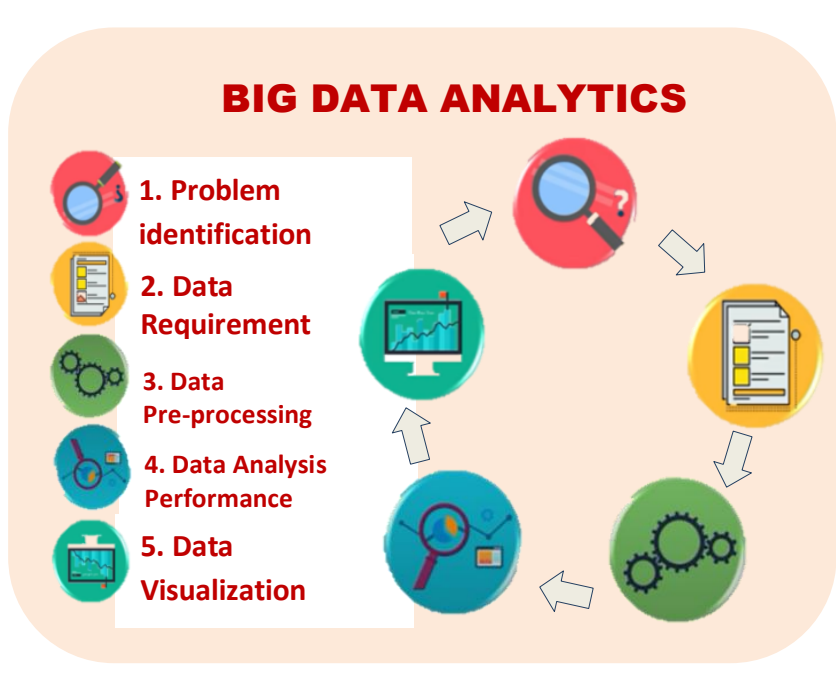

Fig 5: Stages inbig data analytics

\subsection{Cloud-Based Applications}

The dynamics and vibrancy of the day-to-day advancement of technology are occasioned by cloud computing technology. According to [17] who commended the efforts of the industry and academia in bringing out the utility of computing, they in strong terms affirmed that rapid changes seen in a decade ago are occasioned by the services and resources offered by cloud computing. This technology has brought the world into a global village system where information dissemination is more frequent, as a result any new technology will easily be notified as everybody seems to have smart devices that get them hooked up to the cloud and updated with new ideas. This is a technology that has brought the functionality of digital business, webinars for seminars, and virtual classrooms a reality. The service and deployment models cloud computing offers are what actually brought the vibrant dynamism that made the technology acceptable to many. This in essence has changed the face of network and internet technologies. The programming languages used to bring the concept of cloud computinginto a reality are Java, Python, Ruby, PHP, and ASP.NET. 


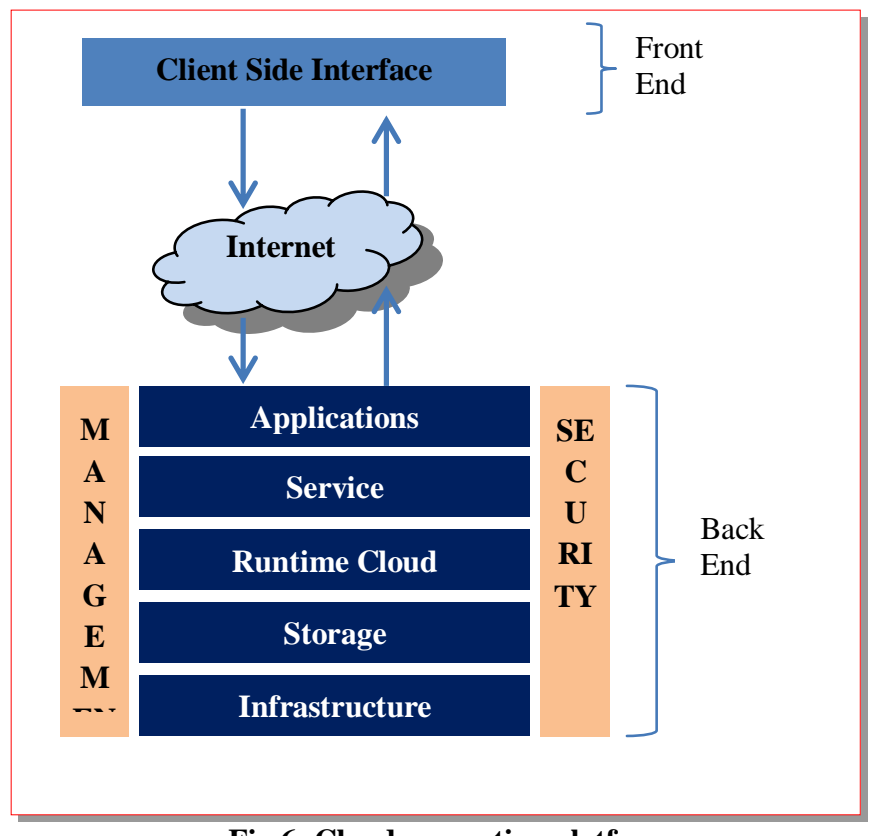

Fig 6: Cloud computing platform

\subsection{Banking Applications}

According to [18], C++ is well used in core-banking systems. Also, its usefulness in the backend programming language is employed by Infosys Finacle. [11] highlighted that the daily processes in banking transactions run into millions of which many tasks happen simultaneously. It is worthy to note that $\mathrm{C}++$ has a reasonable processing speed and multithreading support. In the programming kit of $\mathrm{C}++$, there is a set of templates known as Standard Template Libraries which makes the multithreading support available. All these features when compared to other languages make $\mathrm{C}++$ a preferred programming language in the banking industry.

\subsection{Cyber Security Technology}

As technology is advancing, there is every tendency that security issues will arise and no doubt of security compromises. This issue of course is a very important thing to deal with.So what this technology entails is that it helps to protect the entire computer network from information theft, unauthorized access into the network, and tampering with the integrity of the data. The programming languages useful in this technology are Python, C, C++, JavaScript, and PHP. $\mathrm{SQL}$ is also useful for its role in data management.

\section{CONCLUSION}

Thisstudy has been able to showcase how the instructional codes with problem-solving concept in programming languages have been used to solve societal problems which eventually evolved into various technologies existing today. Thus the use of programming technique and its languages has played prominent role in the evolving technology of the present time.The actual statistics of the rate at which the programming has matched the varying technologies is left for further researches.

\section{REFERENCES}

[1] S. J. Mahmudova, 2018. "Development tendencies of programming languages", Journal of Engineering and Technology, 2018, in press.

[2] O. Ozer, F. Kılıç, "The effect of mobile-assisted language learning environment on EFL students' academic achievement, cognitive load and acceptance of mobile learning tools", EURASIA Journal of Mathematics, Science and Technology Education, 2018, in press.

[3] Edureka. 2019. Top 10 Applications of java programming language.

[4] Edureka. 2020. Top 10 Python applications in the real world you need to know

[5] G. S. Sheikh, N. Islam, “A qualitative study of major programming languages: teaching programming languages to computer science students", Journal of Information \& Communication Technology, 2016, in press.

[6] C. K. Gomathy, "A study on the effect of digital literacy and information management", International Journal of Scientific Research and Review, 2019, in press.

[7] ICF Consulting Services. 2015. Literature review on the impact of digital technology on learning and teaching.

[8] Kholmatova, Z. 2020. Impact of programming languages on energy consumption for mobile devices.

[9] S. Mahmudova, "Features of programming languages and algorithm for calculating the effectiveness" Journal of Information Sciences and Computing Technologies, 2018 , in press.

[10] K. P. N. Reddy, Y. Geyavalli, D. Sujani, S. M. Rajesh, "Comparison of programming languages: review", International Journal of Computer Science \& Communication, 2018, in press.

[11] S. M., Mutongwa, S. Abeka, "A comparative study of C, $\mathrm{C}++, \quad \mathrm{C} \#$ and Java programming languages: Underpinning structure equation modeling, data structures and algorithm", Journal of Scientific and Engineering Research, 2019, in press.

[12] Jiang, L., Rabah, S., Liu, M., and Lai, Y. 2021. Comparative studies of 10 programming languages within 10 diverse criteria.

[13] L. G., Kabari, U. C. Onwuka, "Bradycardia detection using ecg signal processing and MATLAB. EJERS", European Journal of Engineering Research and Science, 2019, in press.

[14] R., Cioffi, M., Travaglioni, G., Piscitelli, A., Petrillo, F. De Felice, "Artificial intelligence and machine learning applications in smart production: progress, trends, and directions",Sustainability, 2020, in press

[15] G., Chawla, S., Bamal,R. Khatana, "Big data analytics for data visualization: review of techniques", International Journal of Computer Applications, 2018, in press.

[16] Burghate, M. 2020. Computer applications for business: the Management Consortium

[17] B., Varghese, R. Buyya, "Next generation cloud computing: new trends and research directions". Future Generation Computing Systems, 2017, in press.

[18] Goel, A. 2020. Uses and application of C++ Language. 